\title{
Improving English listening accuracy of native Japanese by ACE method
}

\author{
Ryota Yasui $^{\mathrm{a}} \quad$ Yutaka Suzuki $^{\mathrm{b}} \quad$ Yasuyuki Satoh $^{\mathrm{a}}$ Osamu Sakata a,* \\ ${ }^{a}$ Tokyo University of Science,6-3-1,Niijuku,Katsusika,Tokyo 125-8585,Japan \\ ${ }^{b}$ University of Yamanashi, 4-3-11 Takeda, Kofu, Yamanashi, Japan \\ Corresponding Author: osakata@ee.kagu.tus.ac.jp
}

\begin{abstract}
Amplitude bandwidth compression/expansion is a method of processing audio signals based on nonlinear amplification characteristics of the human inner ear. This procedure has been confirmed to facilitate listening in people in a noisy environment in addition to assisting the hearing sense of hearing-impaired people.

We herein propose a method of speech processing, mainly English speech, such that Japanese who are not native English speakers can hear the English speech more easily using the ACE method. By changing the frequency division band and amplification parameter based on the speaker's fundamental frequency, individual voices can be processed, unlike when conventional method are used. We conducted a hearing test to validate the proposed method.
\end{abstract}

Keywords: ACE method, Auditory characteristics, nonlinear amplification, fundamental frequency,

MOS evaluations,

\section{Introduction}

The amplitude-bandwidth compression/expansion (ACE) method that processes speech signals using nonlinear amplification characteristics in the human inner ear developed by us is originally intended as a hearing aid for people with hearing impairment ${ }^{(1)}$ It has been confirmed to improve hearing in a noisy environment ${ }^{(2)}$ and provides hearing characteristics close to those of hearing-impaired people and the hearing ability of hearing-impaired people. The effect of the ACE method on hearing aids has been evaluated as positive only when a Japanese native listens to Japanese utterances. However, currently in Japan, opportunities to listen to languages other than Japanese are routine, and voices that require ease of listening are not limited to only Japanese. Therefore, in this study, we examined the improvement method by the ACE method to improve the listening of speech using the English language, which is a language used widely worldwide. Furthermore, we conducted a hearing test using the ACE method on Japanese native hearing users, who constitute the majority of Japanese in the test and confirmed the effect.

\section{Principle}

\subsection{Auditory characteristics of a hearing person under noisy environment}

We show about hearing characteristics between. The relationship between loudness L and sound pressure level I at a pure tone of $1 \mathrm{kHz}$ is shown. Loudness is a psychological amount defined as "attribute of sound presenting a large or small impression," and the units are sone [sone] frequency 1 $\mathrm{kHz}$, sound pressure level $40 \mathrm{~dB}$ SPL, and pure sound of 1 sone. The magnitude of the sound and relational expression ${ }^{(3)}$ are approximated as follows: 


$$
L=k I^{0.3}[\text { sone }],
$$

where $k$ is a coefficient dependent on human hearing. Equation (1) is a power function indicating that the signal in

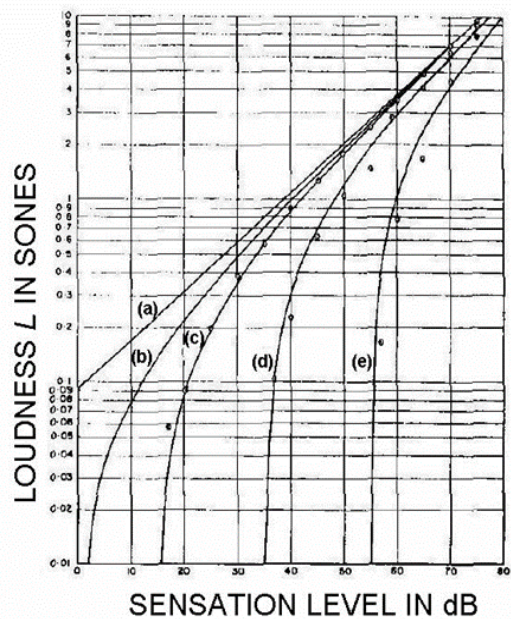

Fig. 1 Listening of a hearing person under noise ${ }^{(6)}$

the sound pressure band up to $100 \mathrm{~dB}$ is compressed to approximately $30 \mathrm{~dB}$ SPL and is close. Hence, it is shown that human beings are louder and louder for smaller sounds. A more accurate relationship between sound pressure level $I$ and loudness $L$ is presented by Lochner and Burger ${ }^{(4)-(7)}$, as shown below:

$$
L=c\left(I^{j}-I_{c}^{j}\right) \text {, }
$$

where $c$ is a linear amplification factor, $j$ is a process of detecting the loudness of a sound, and $I_{c}$ is a human's minimum audible value; additionally, each value changes depending on the frequency band. Therefore, in the region where the intensity $I$ is sufficiently larger than the threshold $I_{c}$, the magnitude is proportional to $I^{j}$. Moreover, if the frequency is changed while the sound intensity is maintained constant for a pure tone, the loudness, which is the size of the sensory sound, changes significantly. Even in a hearing environment, the degree of hearing decreases in noisy environments. The hearing characteristics of a hearing person in a noisy environment are as shown in Fig. ${ }^{(6)}$. The horizontal axis is the sound pressure level [dBSPL] of the stimulation sound, and the vertical axis is the loudness [sone]. The straight line (a) is an approximately straight line when the threshold $I_{c}$ is ignored. Curve (b) represents listening under no noise, and curves (c) (d) (e) indicate the listening when the threshold $I_{c}$ becomes 15, 35, and 55 dBSPL. As shown, it is difficult to hear small sounds in a noisy environment, but loud sounds are almost as audible as when no noise exists. Furthermore, the slope of the curve becomes steeper as the noise level increases; this hearing characteristic can be regarded as similar to sensorineural hearing loss ${ }^{(8)}$. Therefore, it is desirable to relatively amplify small sounds relatively loudly even in a noisy environment under noisy environments.

\subsection{Differences between Japanese and English}

Differences in pronunciation between Japanese and English and phonological phenomena can affect the ease of listening significantly. The assimilation phenomenon, weak phenomenon, dropout phenomenon, etc. are described as examples ${ }^{(9)}$. The weak phenomenon is the pronunciation of a word without an accent. Some English words have different pronunciations depending on whether they are accented; an accented pronunciation is called strong or non-placed pronunciation weak. Furthermore, the combination of weak and strong forms of the rhythm is peculiar in English. In this study, we focused on the negative effects of weakness and strength on the difference in loudness and considered the method of hearing improvement.

\section{Feature of $A C E$ hearing aid processing}

The ACE method is a voice compression method for improving listening from the relationship between the sound pressure level and the loudness of human sound and is expressed as follows:

$$
\mathrm{w}=\operatorname{sgn}(v) k_{a}|v|^{n}
$$

$(v=$ Input voltage[V], $w=$ Output voltage[V], sgn $=$ signum function, $k_{a}=$ Linear amplification factor, $n=$ compression/expansion factor)

Consequently, by amplifying a small sound t relatively large, hearing improvement is expected in a hearingimpaired person and a hearing person in a noisy environment. 
The processing procedure of the ACE method is shown in Fig. 3. The input signal first passes through the BPF to divide the frequency band. Next, nonlinear processing is applied to the divided signals in each band according to Equation (3). It subsequently passes through the BPF again and is generated by the nonlinear processing of the ACE method. Harmonics are removed. Finally, the divided signals are combined and output. In this research, we considered the frequency specific to the voice of each speaker, extracted the fundamental frequency $f_{0}$ from the sound source, and performed band division based on that frequency. In addition, the band was divided into four bands of $1 / 4$ to 8 times (band a), 8 to 16 times (band b), 16 to 32 times (band c), and 32 times to 128 times (band d) of the fundamental frequency. They were subsequently divided and treated with ACE. In the conventional method, the linear amplification coefficient $K_{a}$ and the compression/expansion coefficient $\mathrm{n}$ use the same value in all frequency bands; however, in this study, different linear amplification coefficients $K_{a}$ and compression/ expansion coefficient $\mathrm{n}$ were set for each divided band. In addition, the basic frequency was divided into two at $200 \mathrm{~Hz}$ or less, and the sound source of low-frequency classification was classified into two patterns as male voice and the sound source of high-frequency classification as female voice. Within the two classes of speech, the linear amplification factor $K_{a}$ and the compression/expansion factor $\mathrm{n}$ were considered separately. The male voice sound source strengthened the amplification of the high-frequency band because the low fundamental frequency and female voice sound source compensate for the low-frequency band of sound pressure in the sound source by strengthening the amplification of the low-frequency band owing to the high fundamental frequency. Sound with a relatively small sound pressure difference was created.

\section{Experiments}

\subsection{Experimental method}

A hearing survey was conducted using MOS evaluations (10) (11) using the English sound source before processing and the processed English sound source.

The English sentence used for listening used eight sentences of approximately 15 words that were completed as sentences in one sentence.

After listening to the voice, the subject rated the voice intelligibility on a scale of 5 (1: extremely poor, 2: poor, 3: normal, 4: good, 5: extremely good), and the average value was used as the original voice. We confirmed the change in the ease of hearing by comparing the voice after ACE processing.

\subsection{Result}

The results of the listening experiment are shown in Figure 2 All eight voices indicated improved scores. Hence, the proposed method of this research can be considered effective for improvement in English listening; furthermore, changing

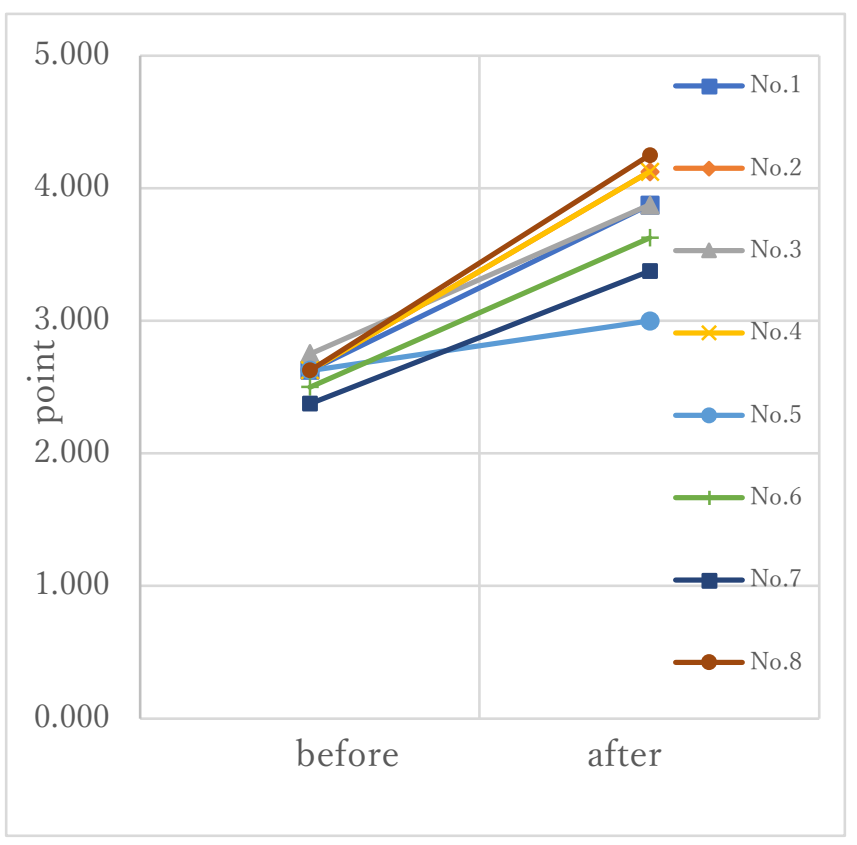

Fig.2 Improvement in listening to each voice

the band division for each speaker, the linear amplification coefficient for each speaker pattern, and the 
compression/expansion coefficient can be regarded as useful.

\section{Conclusion}

In this study, the ACE method that is a method developed by our laboratory to improve both hearing in hearing people with hearing loss and hearing loss by mildly deaf people under a sound environment, was used by native speakers of Japanese who spoke English. A method of changing the frequency band of the speech band division for each speaker based on the fundamental frequency and changing the linear amplification factor and compression/expansion factor for each speaker pattern to improve the ease of listening when listening was proposed. Using the proposed method, we surveyed people who are native speakers of Japanese and evaluated it using a subjective score evaluation method.

The evaluation experiments showed that the proposed method improved audibility. Hence, the method was regarded useful as a hearing aid for English speakers. In addition, by performing speech pattern segmentation and using a division method in which many frequency elements were used in combination with the criteria for band division, performing speech processing consistent with the speech of the speaker resulted in further improvement in audibility.

\section{References}

(1) Y. Suzuki et al., "Evaluation of ACE method based on OSL as hearing aid for sensorineural hearing losses", Journal of Signal Processing,vol.16,no.6,pp.581586,2012

(2) T. Kato et al.,"Universal design of reproduced sound by amplitude-bandwidth compression/expansion Method", Proc.of KEER2007, B-14,2007

(3) B. C. J. Moore,"An Introduction to the Psychology of hearing", Academic Press, pp.127-133,pp.158-159,2003

(4) I.Ishikawa, A.Takeuchi, T.Murano, K.Ohmine and T.kato,"An amplitude-bandwidth expansion method for hearing-aid adjustment", Medical Engineering and
Physics, Vol.27,No.1,pp59-62,2005

(5) C.S.Hllpike and J.D.Hood,"Observations upon the neurological mechanism of the loudness recruitment phenomenon",Acta Oto-Iaryngologica,Vol.50,No36,pp.472-786,1959

(6) J.P.A. Lochner, J. F. Burger, "Form of the Loudness Function in the Presence of Masking Noise", Journal of the Acoustical Society of America,vol.33, pp.1705-1707, 1961

(7) Juhani Parmanen, "Form of the Loudness International Standard ISO 226:2003:AcousticsNormal Equal-Loudness-Level Contours", Open Journal of Acoustics, pp.143-149,2012

(8) B.C.J. Moore et al. "A loudness model for impaired hearing", JASA,vol.106,p.901,1999

(9) Robin C.Scarcella,Rebecca L.Oxford"Second language pronunciation: State of the art in instruction",System, Vol.22, No.2, pp.221-230,1994

(10) Alexander Gutkin, Linne Ha, Martin Jansche, Oddur Kjartansson, Knot Pipatsrisawat, Richard Sproat, "Building Statistical Parametric Multi-speaker Synthesis for Bangladeshi Bangla",Procedia Computer Science, Vol.81,pp.194-200,2016

(11) Robert C. Streij1Stefan Winkler, David S. Hands, "Mean opinion score (MOS) revisited: methods and applications, limitations and alternatives", Multimedia Systems, Volume 22, Issue 2, pp 213-227,2016 